\title{
Convergent losses of TLR5 suggest altered extracellular flagellin detection in four mammalian lineages
}

Virag Sharma ${ }^{1,2,3 \#}$, Felix Walther ${ }^{4}$, Nikolai Hecker ${ }^{1,2,3}$, Heiko Stuckas ${ }^{4}$ and Michael Hiller ${ }^{1,2,3^{*}}$

${ }^{1}$ Max Planck Institute of Molecular Cell Biology and Genetics, Dresden, Germany

${ }^{2}$ Max Planck Institute for the Physics of Complex Systems, Dresden, Germany

${ }^{3}$ Center for Systems Biology Dresden, Germany

${ }^{4}$ Senckenberg Natural History Collections Dresden, Senckenberg - Leibniz Institution for Biodiversity and Earth System Research

*To whom correspondence should be addressed:

Michael Hiller

Computational Biology and Evolutionary Genomics, Max Planck Institute of Molecular Cell Biology and Genetics \& Max Planck Institute for the Physics of Complex Systems, Dresden, Germany.

Tel: +493512102781

Fax: +493512101209

Email: hiller@mpi-cbg.de

\# current affiliations: CRTD-DFG Center for Regenerative Therapies Dresden, Carl Gustav Carus Faculty of Medicine, Technische Universität Dresden, Dresden; Paul Langerhans Institute Dresden (PLID) of the Helmholtz Center Munich at University Hospital Carl Gustav Carus and Faculty of Medicine, Technische Universität Dresden, Dresden; German Center for Diabetes Research (DZD), Munich, Neuherberg, Germany.

Running title: Convergent losses of TLR5 in mammals

Keywords: innate immunity, toll-like receptors, TLR5, flagellin, gene loss 


\section{Abstract}

Toll-like receptors (TLRs) play an important role for the innate immune system by detecting pathogen-associated molecular patterns. TLR5 encodes the major extracellular receptor for bacterial flagellin and frequently evolves under positive selection, consistent with coevolutionary arms races between the host and pathogens. Furthermore, TLR5 is inactivated in several vertebrates and a TLR5 stop codon polymorphism is widespread in human populations. Here, we analyzed the genomes of 120 mammals and discovered that TLR5 is convergently lost in four independent lineages, comprising guinea pigs, Yangtze river dolphin, pinnipeds, and pangolins. Validated inactivating mutations, absence of protein-coding transcript expression, and relaxed selection on the TLR5 remnants confirm these losses. PCR analysis further confirmed the loss of TLR5 in the pinniped stem lineage. Finally, we show that TLR11, encoding a second extracellular flagellin receptor, is also absent in these four lineages. Independent losses of TLR5 and TLR11 suggests that a major pathway for detecting flagellated bacteria is not essential for different mammals and predicts an impaired capacity to sense extracellular flagellin. 


\section{Introduction}

Toll-like receptor (TLR) proteins are an important component of the innate immune system (Vijay 2018). TLRs comprise a group of membrane-bound pattern recognition receptors and detect pathogen-associated molecular patterns such as bacterial lipopolysaccharides, peptidoglycans, double-stranded RNA or flagellin (Vijay 2018). Extracellular flagellin is recognized by TLR5 (toll-like receptor 5) (Hayashi, et al. 2001; Mizel, et al. 2003; Smith, et al. 2003). Upon binding its ligand, TLR5 stimulates the expression of proinflammatory, antibacterial and stress-related genes (Yu, et al. 2003; Vijay-Kumar, et al. 2008), and hence plays an important role for recognizing pathogenic flagellated bacteria such as Salmonella (Murthy, et al. 2004). The flagellin sensor function of TLR5 is likely conserved among vertebrates given that TLR5 of chicken, Anole lizard and rainbow trout also recognizes bacterial flagellin (Tsujita, et al. 2004; lqbal, et al. 2005; Voogdt, et al. 2016). Importantly, TIr5 knockout mice do not show an immune response to injected flagellin (Feuillet, et al. 2006; Uematsu, et al. 2006; Vijay-Kumar, et al. 2007), suggesting that TIr5 is the main extracellular flagellin sensor.

Coevolutionary arms races between pathogens and the host likely explain why pathogensensing immune genes frequently evolve under positive selection (Sackton, et al. 2007). Consistently, the TLR5 gene experienced positive selection in many primate, mammal and bird lineages, and recurrent positive selection was often detected for sites located near the ligand binding site (Wlasiuk, et al. 2009; Alcaide, et al. 2011; Areal, et al. 2011; Smith, et al. 2012; Vinkler, et al. 2014; Velova, et al. 2018). Interestingly, despite its conserved role in detecting flagellin, previous studies revealed that TLR5 is inactivated (lost) in several independent bird lineages (Alcaide, et al. 2011; Bainova, et al. 2014; Velova, et al. 2018), in tuatara and in clownfish (Liu, et al. 2019). Furthermore, a dominant negative TLR5 polymorphism that introduces a premature stop codon is present in $\sim 4 \%$ of human individuals and has reached a frequency of $>20 \%$ in some populations (Hawn, et al. 2003; Barreiro, et al. 2009; Wlasiuk, et al. 2009). Therefore, the question arises whether inactivation of TLR5 also occurred in non-human mammals. Here, by analyzing the genomes of 120 mammalian species, we show that TLR5 is lost in four independent mammalian lineages, comprising guinea pigs, Yangtze river dolphin, pinnipeds, and pangolins. 


\section{Results}

We applied a previously developed approach (Sharma, et al. 2018a) to screen for geneinactivating mutations in TLR5 using a genome alignment of 120 placental mammals (Supplementary Table 1) (Hecker, et al. 2020). This screen revealed that the single coding exon of TLR5 exhibits stop codon mutations, smaller frameshifting insertions or deletions, or larger deletions in the genome assemblies of 17 species belonging to 14 distinct lineages (gorilla, orangutan, guinea pig, rabbit, pig, alpaca, Yangtze river dolphin, bighorn sheep, Weddell seal, Hawaiian monk seal, walrus, Malayan and Chinese pangolins, little brown bat, elephant, armadillo). Since we have previously shown that apparent gene-inactivating mutations can sometimes be sequencing or assembly errors (Hecker, et al. 2017; Sharma, et al. 2018b; Hecker, et al. 2019a; Huelsmann, et al. 2019), we carefully validated the correctness of all such putative mutations and performed additional analyses to establish which of these 17 species truly lost TLR5.

First, we used available raw sequencing read data to validate stop codon and smaller frameshifting insertion/deletion mutations. This revealed that frameshifting insertions and deletions that are apparent in the orangutan, rabbit, alpaca, bighorn sheep, little brown bat, elephant and armadillo assemblies are in fact base errors in the assemblies as aligning raw sequencing reads do not validate these putative mutations (Supplementary Figure $1 \mathrm{~A}-\mathrm{J}$ ). Similarly, sequencing reads show that $1 \mathrm{bp}$ frameshifting deletions in the gorilla (gorGor5) and pig (susScr11) genomes that are assembled from PacBio long sequencing reads (Gordon, et al. 2016; Warr, et al. 2019) are base errors (Supplementary Figure 2). Furthermore, these two base errors are not present in previous assemblies of gorilla (gorGor3) and pig (susScr3), and therefore are likely due to uncorrected errors present in raw PacBio reads (Watson, et al. 2019). In contrast to these nine species, all frameshifts and stop codon mutations present in the domestic guinea pig, Weddell seal, and Pacific walrus were confirmed by several sequencing reads and we found no support for the ancestral, non-inactivating allele (Figure 1A, Supplementary Table 2).

Second, we analyzed the pairwise genome alignments between human and guinea pig, river dolphin, pinnipeds, and pangolins. This confirmed that the remnants of TLR5 occur in a genomic locus with conserved gene synteny (Supplementary Figure 3). Furthermore, we found no evidence for the presence of a functional duplicated TLR5 copy in these 
genomes. Together, this shows that the observed losses of TLR5 are not artifacts arising from aligning paralogous or processed pseudogenes.

Third, since no raw sequencing reads were available to confirm the four TLR5-inactivating mutations observed in the Yangtze river dolphin assembly (Figure 1A), we made use of the recently assembled genomes of the Amazon river and La Plata dolphins, which are sister species of the Yangtze river dolphin (Geisler, et al. 2011), and investigated whether any of these inactivating mutations are shared. Indeed, we found that the second stop codon mutation is shared between all three river dolphin species (Figure $1 \mathrm{~A}$ ). The presence of the same mutation in independently sequenced and assembled genomes makes it extremely unlikely that this mutation is a base error. Instead, this shared mutation strongly indicates that TLR5 was already lost in the common ancestor of the three river dolphin species. We also found additional species-specific frameshifting mutations in the Amazon river and La Plata dolphins, which further support that TLR5 is lost in these two species as well (Supplementary Figure 4). Similarly, while no sequencing reads are available to confirm mutations in the Hawaiian monk seal assembly, we found that six of seven observed mutations are shared with the Weddell seal (Figure 1A), providing strong support that TLR5 is lost in the Hawaiian monk seal.

To investigate whether TLR5 loss is specific to the domestic guinea pig or whether this gene is also lost in wild guinea pig species, we analyzed the TLR5 locus in the genomes of two additional guinea pigs which are not a part of our genome alignment - Brazilian and Montane guinea pigs. As shown in Figure 1A, both stop codons and the 2 bp deletion are shared between all three guinea pig species. Additionally, the large deletion that removes most of the TLR5 coding exon has the same breakpoints in all three guinea pig species (Figure 1B), which makes an assembly error highly unlikely and suggests that this deletion already occurred in their common ancestor. Furthermore, all three guinea pig species share larger structural rearrangements in this locus including several duplications that happened after the deletion (Supplementary Figure 5).

TLR5 is entirely deleted in the genomes of the Chinese and Malayan pangolin (Figure 1A). To further investigate whether this deletion could be an assembly error, we generated alignment chains for the Tree pangolin, another recently sequenced pangolin species that was not included in our alignment. We found that TLR5 is also deleted in the tree pangolin and the deletion break points are shared between all three pangolins (Figure 
$1 \mathrm{C})$, which strongly suggests that the gene deletion already occurred in the common ancestor of the three pangolin species.

The three pinniped species in our data set share several frameshifting deletions (Figure 1A). Since these three species represent only the two of the three basal pinniped lineages, Phocidae (Weddell seal, Hawaiian monk seal) and Odobenidae (walrus), we investigated whether TLR5 is also lost in the third pinniped lineage Otariidae. To this end, we used PCR to amplify TLR5 from tissue samples of the brown fur seal, an otariid seal. Sequencing confirmed that the $25 \mathrm{bp}$ and $17 \mathrm{bp}$ deletion mutations are also present in the brown fur seal (Figure 1D). Furthermore, we found the same mutations in the Antarctic fur seal genome (Supplementary Figure 6). Together, the presence of inactivating mutations shared between Otariidae, Phocidae and Odobenidae suggests that TLR5 loss already occurred in the pinniped stem lineage at least 26 Mya (the estimated split of pinnipeds according to TimeTree (Kumar, et al. 2017b)).

Fourth, we used available transcriptomic data to explore whether there is still expression at the TLR5 locus in the gene loss species. While we found no evidence for expression in the guinea pig and pangolin (Supplementary Figures 7, 8), clear expression at the TLR5 locus was observed in Weddell seal (Supplementary Figure 9). Importantly, RNA-seq reads exhibit the frameshifting deletions that are present in the Weddell seal genome assembly, showing that these transcripts cannot encode a functional TLR5 protein. Consistent with previous findings (Sadier, et al. 2018), this suggests that the remnants of a lost coding gene can remain to be transcribed, in our case for at least $26 \mathrm{My}$.

Finally, we used RELAX (Wertheim, et al. 2015) to test whether TLR5 evolves under relaxed selection in pinnipeds and the river dolphin where a substantial portion of the gene is still present. We did not apply RELAX to pangolins and guinea pigs where $>80 \%$ of the gene is deleted. Further supporting TLR5 loss in pinnipeds and Yangtze river dolphin, we found significant evidence for relaxed selection in both lineages $(\mathrm{Ka} / \mathrm{Ks}=$ 1.17, $\mathrm{P}$-value $=0.0002$ for Yangtze river dolphin and $\mathrm{Ka} / \mathrm{Ks}=0.78, \mathrm{P}$-value $=0.0009$ for pinnipeds) (Supplementary Table 3).

With several lines of evidence ranging from signatures of relaxed selection and the presence of validated inactivating mutations, we conclusively show that TLR5 has been lost in four independent mammalian lineages. These mutations either delete the majority 
or the entire gene (guinea pigs, pangolins) or severely affect the reading frame and domains required for protein function (Figure 1A), suggesting that the remnants of TLR5 cannot encode a functional flagellin-recognition receptor anymore.

Apart from TLR5, mouse TIr11 has been shown to bind flagellin (Mathur, et al. 2012), even though the TIr11-flagellin interaction is restricted to acidic conditions (Hatai, et al. 2016). Therefore, we considered the possibility that TLR5 loss may be compensated by the presence of TLR11, and investigated whether the TLR5-loss species possess an intact TLR11 gene. However, we found that TLR11 is most likely lost in the genomes of guinea pig, river dolphin, pinnipeds, and pangolins (Supplementary Figure 10), indicating that this gene cannot compensate for the loss of the flagellin-sensing TLR5.

\section{Discussion}

Here, we show that TLR5, the gene encoding the major extracellular flagellin receptor, has been convergently lost four times in mammalian evolution. Our study highlights the importance of carefully validating putative gene-inactivating mutations to detect real gene loss events. All frameshifts detected by our gene loss detection method (Sharma, et al. 2018a) are really present in the respective assemblies, corroborating the high accuracy of our method; however, our sequencing read validation revealed that many of these frameshifts are actually base errors in genome assemblies that were generated from short or long sequencing reads. While current genome assembly efforts often focus on generating highly-contiguous assemblies (Miga, et al. 2019), our observations suggest that assembly base accuracy is potentially an underappreciated issue. Indeed, nearperfect assembly base accuracy would be of great importance to automatically analyze genomic data without the need to manually validate putative mutations.

Based on human and mouse studies (Hawn, et al. 2003; Feuillet, et al. 2006; Uematsu, et al. 2006; Vijay-Kumar, et al. 2007), loss of TLR5 is predicted to severely impair the capacity to sense extracellular flagellin, in particular because all four TLR5-loss lineages also lack an intact TLR11 gene that may have acted as a compensating factor. An intriguing question is why the evolutionary inactivation of both extracellular flagellin receptors does apparently not have deleterious consequences for guinea pigs, river dolphins, pinnipeds, and pangolins. A potential explanation could be that alternative 
pathways for flagellin detection compensate for the loss of TLR5 and TLR11. For example, we recently described mammals that lost several human disease-associated genes but do not show the deleterious, disease-resembling phenotypes (Sharma, et al. 2019), which suggests the existence of either functionally-redundant genes or compensatory mechanisms that may have permitted losses of human disease genes in other mammals.

Whether the "natural TLR5 knockout" mammals retain a capacity to sense extracellular flagellin could be tested experimentally. While research in river dolphins, pangolins and pinnipeds is generally impractical, guinea pigs have been used in biomedical research including studies of asthma, tuberculosis, Zika virus and other infectious diseases (Meurs, et al. 2006; Clark, et al. 2014; Kumar, et al. 2017a), because many aspects of guinea pig physiology and immunology are more similar to humans than to mouse or rat (PadillaCarlin, et al. 2008). Thus, in addition to targeted research in mouse, guinea pigs could be used as another rodent model to study the consequences of natural TLR5 knockout mammals.

Our findings raise questions of which evolutionary and ecological factors are associated or drove the evolutionary losses of TLR5 in non-human mammals. TLR5 also plays an important role in establishing and maintaining a healthy gut microbiome (Vijay-Kumar, et al. 2010; Fulde, et al. 2018) and gut microbiome composition is influenced by diet (Delsuc, et al. 2014; Youngblut, et al. 2019), raising the possibility that dietary switches could be one of the involved factors. However, while all four mammalian TLR5-loss lineages are dietary specialists, they specialize in different diets, ranging from carnivorous (river dolphins, pinnipeds), herbivorous (guinea pigs) to myrmecophagous (pangolins that feed on termites and ants) diets. This indicates that specialization to a particular diet was likely not a major factor behind the evolutionary losses of this gene. Furthermore, many other mammals convergently specialized on carnivorous, herbivorous and myrmecophagous diets without losing TLR5.

In general, loss of a gene can be due to relaxed selection to preserve its function or provide an evolutionary advantage (Albalat, et al. 2016; Sharma, et al. 2018a; Hecker, et al. 2019b; Huelsmann, et al. 2019). Interestingly, in humans, the TLR5 stop codon mutation is associated with both positive and negative consequences. On the one hand, TLR5 inactivation is associated with an increased susceptibility to Legionella-induced 
pneumonia and recurrent urinary tract infections, and constitutes a risk factor for type 2 diabetes (Hawn, et al. 2003; Hawn, et al. 2009; Al-Daghri, et al. 2013). On the other hand, TLR5 inactivation is associated with an improved survival in melioidosis patients (West, et al. 2013), and in certain ethnic groups with a protection from systemic lupus erythematosus and Crohn's disease (Hawn, et al. 2005; Gewirtz, et al. 2006). This indicates that inactivating TLR5 can also have a protective effect against the immune system dysregulation that is involved in these diseases. Such beneficial effects may explain why this stop codon mutation has reached a relatively high frequency of $>4 \%$ in many human populations, although signatures of recent adaptive evolution have not been detected (Barreiro, et al. 2009; Wlasiuk, et al. 2009). Since an inflammatory response represents a cost-benefit trade-off that is often optimized for a particular environment (Okin, et al. 2012), adjusting inflammatory responses can be an advantageous evolutionary strategy for species occupying specific environmental niches, exemplified by an attenuated inflammatory response to lipopolysaccharides in blood of deep diving seals (Bagchi, et al. 2018). Thus, it remains to be elucidated whether the TLR5 losses in the four non-human mammalian lineages were due to relaxed selection or offered an evolutionary advantage. 


\section{Materials and Methods}

\section{Investigating and validating losses of TLR5}

We used our gene loss detection method (Sharma, et al. 2018a) to investigate whether gene-inactivating mutations (frameshifting insertions/deletions, premature stop codon mutations, splice site disrupting mutations, large deletions) occur in TLR5 in mammals. Since the two TLR5 transcripts in human GENCODE version 32 (Harrow, et al. 2012) annotate an identical single coding exon and only differ in 5' UTR exons, we focused on this single coding exon. We used human as the reference species and initially considered a total of 119 non-human mammals that are part of a 120-mammal genome alignment (Hecker, et al. 2020). The TLR5 sequence provided by this alignment was re-aligned with CESAR (Codon Exon Structure Aware Realigner) (Sharma, et al. 2016; Sharma, et al. 2017 ) to avoid spurious frameshifts due to alignment ambiguities. Assembly gaps that overlap parts of TLR5 in two species were not taken as evidence for gene loss (see Supplementary Figure 11).

Validation of small putative inactivating mutations was done as described before (Jebb, et al. 2018; Sharma, et al. 2018b). Briefly, we extracted a 50 bp genomic context surrounding a mutation, aligned these sequences against sequencing reads stored in SRA (Kodama, et al. 2012) (accession numbers for each species are listed in Supplementary Table 1), and counted how many reads support the inactivating mutation or the ancestral non-gene-inactivating allele. To investigate large deletions, we visualized and inspected pairwise alignment chains (Kent, et al. 2003) in the UCSC genome browser (Haeussler, et al. 2019). In addition, alignment chains were used to investigate whether remnants of TLR5 occur in a context of conserved gene order and to exclude the possibility that a functional TLR5 copy exists elsewhere in these assemblies.

Gene-inactivating mutations that are shared between related species are strong evidence against assembly base errors and indicate that gene loss is fixed in the respective clade (Hecker, et al. 2019b; Huelsmann, et al. 2019; Sharma, et al. 2019). Therefore, in addition to the 119 non-human mammals included in the 120-mammal genome alignment, we analyzed TLR5 in six additional mammals that are close sister species to TLR5-loss species (two river dolphins, two guinea pigs, tree pangolin and Antarctic fur seal; NCBI assembly accession numbers are listed in Supplementary Table 1). For these six species, 
we computed sensitive pairwise alignment chains to human (hg38) using lastz (Harris 2007) (alignment parameters $\mathrm{K}=2400, \mathrm{~L}=3000, \mathrm{Y}=9400, \mathrm{H}=2000$, default scoring matrix), axtChain (Kent, et al. 2003) (linearGap=loose, otherwise default parameters), and RepeatFiller (Osipova, et al. 2019) (default parameters). The presence of shared inactivating mutations was manually confirmed.

\section{Relaxed selection}

We used RELAX (Wertheim, et al. 2015) to determine whether the remaining TLR5 coding sequence evolves under relaxed selection in the Yangtze river dolphin and in pinnipeds. To this end, we first extracted the coding sequence of TLR5 from the 120mammal alignment. Then, we generated pairwise alignments between the human coding sequence and the sequence corresponding to each of the 119 mammals using CESAR. These pairwise alignments were combined into a multiple sequence alignment using mafjoin (Kielbasa, et al. 2011). The multiple alignment was further post-processed by removing alignment columns that contained frameshifting insertions and by substituting in-frame stop codons by NNN. We ran the test for relaxed selection twice and specified either the Yangtze river dolphin branch or the branch leading to pinnipeds as the foreground. All other branches leading to species that did not lose TLR5 were specified as background.

\section{Analysis of transcriptomic data}

RNA-seq data of guinea pig brain (SRP017611) (Fushan, et al. 2015), lung (SRP040447) (Davidsen, et al. 2014), ovaries and testis (SRP104222) (Bens, et al. 2018), Malayan pangolin cerebrum and lung (SRP064341) (Mohamed Yusoff, et al. 2016), small intestine, large intestine and stomach (SRP101596 and SRP156258, Guangdong Institute of Applied Biological Resources), and Weddell seal brain, lung, placenta, and testis (SRP200409, Broad Institute) was downloaded from the Sequence Read Archive (SRA) (Kodama, et al. 2012). Next, fastq-dump was used to process the initial read data using parameters for removing technical reads (skip-technical), filtering low-quality reads (readfilter pass), removing tags (clip), converting data into base space (dumpbase), keeping read identifiers (readids) and splitting paired-end reads into separate files (split-files). Reads were mapped to the corresponding genomes with STAR (version 2.4.2a) (Dobin, et al. 2013). For Malayan pangolin, we adjusted the number of bins for genomic indexes (genomeChrBinNbits: 15). Reads were mapped separately per run as paired-end reads with parameters limiting the ratio of mismatches per mapped read 
(outFilterMismatchNoverLmax=0.04) and limiting the number of mapping locations per read (outFilterMultimapNmax $=20$ ), and otherwise defaults. To visualize gene expression in the UCSC Genome Browser (Haeussler, et al. 2019), we processed the mapped reads with BEDtools (Quinlan, et al. 2010) and bedGraphToBigWig (Kent, et al. 2010).

\section{PCR analysis of TLR5 in the brown fur seal}

Tissue samples of the brown fur seal (Arctocephalus pusillus; Pinnipedia, Otariidae) were obtained from Senckenberg Natural History Collections Dresden, Germany (collection ID: MTD-TB 250) representing a specimen from the Zoological Garden Leipzig (Germany). DNA was extracted using the innuPrep DNA Mini Kit (Analytik Jena, Jena, Germany) following the manufacturer's instructions (protocol for DNA isolation from tissue samples or rodent tails) except that tissue lysis was performed overnight and DNA was precipitated in two steps using 50 $\mu$ l elution buffer. Standard PCR reactions were performed in a total volume of $20 \mu \mathrm{l}$ using 10-20ng DNA, one unit DFS-Taq polymerase (Bioron, Ludwigshafen, Germany) in the recommended buffer, $3.5 \mathrm{mM} \mathrm{MgCl}, 0.2 \mathrm{mM}$ of each dNTP (Fermentas, St. Leon-Rot, Germany), and $0.37 \mu \mathrm{M}$ of each primer listed in Supplementary Table 4. PCR amplification of target TLR5 regions was performed with primer combinations shown in Supplementary Figure 12, using 35 PCR cycles with denaturation at $94^{\circ} \mathrm{C}\left(15 \mathrm{sec}\right.$ but $3 \mathrm{~min}$ for the first cycle), annealing at $63^{\circ} \mathrm{C}(20-30$ sec), and extension at $72^{\circ} \mathrm{C}(1: 30 \mathrm{~min}$ but $7 \mathrm{~min}$ for the last cycle). PCR products were Sanger sequenced in both directions after enzymatic clean-up with ExoSAP-IT (USB Europe $\mathrm{GmbH}$, Staufen, Germany), cycle sequencing using the PCR primers, the BigDye Terminator v3.1 Cycle Sequencing Kit (Applied Biosystems) and an ABI 3130xI Genetic Analyser (Applied Biosystems, Foster City, CA, USA).

\section{Data availability}

All analyzed genome assemblies and sequencing read data is publicly available on NCBI and SRA (accession numbers listed in Supplementary Table 1). The multiple genome alignment is available at https://bds.mpicbg.de/hillerlab/120MammalAlignment/Human120way/. The TLR5 sequence of the brown fur seal has been submitted to NCBI (accession number pending).

\section{Competing interests}

The authors have no competing interests. 


\section{Acknowledgment}

We thank the genomics community for sequencing and assembling the genomes and the UCSC genome browser group for providing software and genome annotations. We also thank Bogdan Kirilenko for computing river dolphin genome alignments, and the Computer Service Facilities of the MPI-CBG and MPI-PKS for their support. This work was supported by the Max Planck Society, the German Research Foundation (HI 1423/31) and the Leibniz Association (SAW-2016-SGN-2).

\section{References}

Al-Daghri NM, Clerici M, Al-Attas O, Forni D, Alokail MS, Alkharfy KM, Sabico S, Mohammed AK, Cagliani R, Sironi M. 2013. A nonsense polymorphism (R392X) in TLR5 protects from obesity but predisposes to diabetes. J Immunol 190:3716-3720.

Albalat R, Canestro C. 2016. Evolution by gene loss. Nat Rev Genet 17:379-391.

Alcaide M, Edwards SV. 2011. Molecular evolution of the toll-like receptor multigene family in birds. Mol Biol Evol 28:1703-1715.

Areal H, Abrantes J, Esteves PJ. 2011. Signatures of positive selection in Toll-like receptor (TLR) genes in mammals. BMC evolutionary biology 11:368.

Bagchi A, Batten AJ, Levin M, Allen KN, Fitzgerald ML, Huckstadt LA, Costa DP, Buys ES, Hindle AG. 2018. Intrinsic anti-inflammatory properties in the serum of two species of deep-diving seal. J Exp Biol 221.

Bainova H, Kralova T, Bryjova A, Albrecht T, Bryja J, Vinkler M. 2014. First evidence of independent pseudogenization of toll-like receptor 5 in passerine birds. Dev Comp Immunol 45:151-155.

Barreiro LB, Ben-Ali M, Quach H, Laval G, Patin E, Pickrell JK, Bouchier C, Tichit M, Neyrolles O, Gicquel B, et al. 2009. Evolutionary dynamics of human Toll-like receptors and their different contributions to host defense. PLoS genetics 5:e1000562.

Bens M, Szafranski K, Holtze S, Sahm A, Groth M, Kestler HA, Hildebrandt TB, Platzer M. 2018. Naked molerat transcriptome signatures of socially suppressed sexual maturation and links of reproduction to aging. BMC Biol 16:77.

Clark S, Hall Y, Williams A. 2014. Animal models of tuberculosis: Guinea pigs. Cold Spring Harb Perspect Med 5:a018572.

Davidsen PK, Herbert JM, Antczak P, Clarke K, Ferrer E, Peinado VI, Gonzalez C, Roca J, Egginton S, Barbera JA, et al. 2014. A systems biology approach reveals a link between systemic cytokines and skeletal muscle energy metabolism in a rodent smoking model and human COPD. Genome Med 6:59.

Delsuc F, Metcalf JL, Wegener Parfrey L, Song SJ, Gonzalez A, Knight R. 2014. Convergence of gut microbiomes in myrmecophagous mammals. Mol Ecol 23:1301-1317.

Dobin A, Davis CA, Schlesinger F, Drenkow J, Zaleski C, Jha S, Batut P, Chaisson M, Gingeras TR. 2013. STAR: ultrafast universal RNA-seq aligner. Bioinformatics 29:15-21. 
Feuillet V, Medjane S, Mondor I, Demaria O, Pagni PP, Galan JE, Flavell RA, Alexopoulou L. 2006. Involvement of Toll-like receptor 5 in the recognition of flagellated bacteria. Proc Natl Acad Sci U S A 103:12487-12492.

Fulde M, Sommer F, Chassaing B, van Vorst K, Dupont A, Hensel M, Basic M, Klopfleisch R, Rosenstiel P, Bleich $A$, et al. 2018. Neonatal selection by Toll-like receptor 5 influences long-term gut microbiota composition. Nature 560:489-493.

Fushan AA, Turanov AA, Lee SG, Kim EB, Lobanov AV, Yim SH, Buffenstein R, Lee SR, Chang KT, Rhee H, et al. 2015. Gene expression defines natural changes in mammalian lifespan. Aging Cell 14:352-365.

Geisler JH, McGowen MR, Yang G, Gatesy J. 2011. A supermatrix analysis of genomic, morphological, and paleontological data from crown Cetacea. BMC evolutionary biology 11:112.

Gewirtz AT, Vijay-Kumar M, Brant SR, Duerr RH, Nicolae DL, Cho JH. 2006. Dominant-negative TLR5 polymorphism reduces adaptive immune response to flagellin and negatively associates with Crohn's disease. Am J Physiol Gastrointest Liver Physiol 290:G1157-1163.

Gordon D, Huddleston J, Chaisson MJ, Hill CM, Kronenberg ZN, Munson KM, Malig M, Raja A, Fiddes I, Hillier LW, et al. 2016. Long-read sequence assembly of the gorilla genome. Science 352:aae0344.

Haeussler M, Zweig AS, Tyner C, Speir ML, Rosenbloom KR, Raney BJ, Lee CM, Lee BT, Hinrichs AS, Gonzalez JN, et al. 2019. The UCSC Genome Browser database: 2019 update. Nucleic acids research 47:D853-D858.

Harris RS. 2007. Improved pairwise alignment of genomic DNA. [The Pennsylvania State University.

Harrow J, Frankish A, Gonzalez JM, Tapanari E, Diekhans M, Kokocinski F, Aken BL, Barrell D, Zadissa A, Searle $S$, et al. 2012. GENCODE: the reference human genome annotation for The ENCODE Project. Genome research 22:1760-1774.

Hatai H, Lepelley A, Zeng W, Hayden MS, Ghosh S. 2016. Toll-Like Receptor 11 (TLR11) Interacts with Flagellin and Profilin through Disparate Mechanisms. PLoS One 11:e0148987.

Hawn TR, Scholes D, Li SS, Wang H, Yang Y, Roberts PL, Stapleton AE, Janer M, Aderem A, Stamm WE, et al. 2009. Toll-like receptor polymorphisms and susceptibility to urinary tract infections in adult women. PLoS One 4:e5990.

Hawn TR, Verbon A, Lettinga KD, Zhao LP, Li SS, Laws RJ, Skerrett SJ, Beutler B, Schroeder L, Nachman A, et al. 2003. A common dominant TLR5 stop codon polymorphism abolishes flagellin signaling and is associated with susceptibility to legionnaires' disease. J Exp Med 198:1563-1572.

Hawn TR, Wu H, Grossman JM, Hahn BH, Tsao BP, Aderem A. 2005. A stop codon polymorphism of Tolllike receptor 5 is associated with resistance to systemic lupus erythematosus. Proc Natl Acad Sci U S A 102:10593-10597.

Hayashi F, Smith KD, Ozinsky A, Hawn TR, Yi EC, Goodlett DR, Eng JK, Akira S, Underhill DM, Aderem A. 2001. The innate immune response to bacterial flagellin is mediated by Toll-like receptor 5 . Nature 410:1099-1103.

Hecker N, Hiller M. 2020. A genome alignment of 120 mammals highlights ultraconserved element variability and placenta-associated enhancers. Gigascience 9.

Hecker N, Lachele U, Stuckas H, Giere P, Hiller M. 2019a. Convergent vomeronasal system reduction in mammals coincides with convergent losses of calcium signalling and odorant-degrading genes. Mol Ecol 28:3656-3668. 
Hecker N, Sharma V, Hiller M. 2019b. Convergent gene losses illuminate metabolic and physiological changes in herbivores and carnivores. Proc Natl Acad Sci U S A 116:3036-3041.

Hecker N, Sharma V, Hiller M. 2017. Transition to an Aquatic Habitat Permitted the Repeated Loss of the Pleiotropic KLK8 Gene in Mammals. Genome Biol Evol 9:3179-3188.

Huelsmann M, Hecker N, Springer MS, Gatesy J, Sharma V, Hiller M. 2019. Genes lost during the transition from land to water in cetaceans highlight genomic changes associated with aquatic adaptations. Sci Adv 5:eaaw6671.

Iqbal M, Philbin VJ, Withanage GS, Wigley P, Beal RK, Goodchild MJ, Barrow P, McConnell I, Maskell DJ, Young J, et al. 2005. Identification and functional characterization of chicken toll-like receptor 5 reveals a fundamental role in the biology of infection with Salmonella enterica serovar typhimurium. Infect Immun 73:2344-2350.

Jebb D, Hiller M. 2018. Recurrent loss of HMGCS2 shows that ketogenesis is not essential for the evolution of large mammalian brains. Elife 7:e38906.

Kent WJ, Baertsch R, Hinrichs A, Miller W, Haussler D. 2003. Evolution's cauldron: duplication, deletion, and rearrangement in the mouse and human genomes. Proc Natl Acad Sci U S A 100:11484-11489.

Kent WJ, Zweig AS, Barber G, Hinrichs AS, Karolchik D. 2010. BigWig and BigBed: enabling browsing of large distributed datasets. Bioinformatics 26:2204-2207.

Kielbasa SM, Wan R, Sato K, Horton P, Frith MC. 2011. Adaptive seeds tame genomic sequence comparison. Genome research 21:487-493.

Kodama Y, Shumway M, Leinonen R, International Nucleotide Sequence Database C. 2012. The Sequence Read Archive: explosive growth of sequencing data. Nucleic acids research 40:D54-56.

Kumar M, Krause KK, Azouz F, Nakano E, Nerurkar VR. 2017a. A guinea pig model of Zika virus infection. Virol J 14:75.

Kumar S, Stecher G, Suleski M, Hedges SB. 2017b. TimeTree: A Resource for Timelines, Timetrees, and Divergence Times. Mol Biol Evol 34:1812-1819.

Liu G, Zhang H, Zhao C, Zhang H. 2019. Evolutionary history of the Toll-like receptor gene family across vertebrates. Genome Biol Evol.

Mathur R, Oh H, Zhang D, Park SG, Seo J, Koblansky A, Hayden MS, Ghosh S. 2012. A mouse model of Salmonella typhi infection. Cell 151:590-602.

Meurs H, Santing RE, Remie R, van der Mark TW, Westerhof FJ, Zuidhof AB, Bos IS, Zaagsma J. 2006. A guinea pig model of acute and chronic asthma using permanently instrumented and unrestrained animals. Nat Protoc 1:840-847.

Miga KH, Koren S, Rhie A, Vollger MR, Gershman A, Bzikadze A, Brooks S, Howe E, Porubsky D, Logsdon $\mathrm{GA}$, et al. 2019. Telomere-to-telomere assembly of a complete human X chromosome. BioRxiv:735928.

Mizel SB, West AP, Hantgan RR. 2003. Identification of a sequence in human toll-like receptor 5 required for the binding of Gram-negative flagellin. The Journal of biological chemistry 278:23624-23629.

Mohamed Yusoff A, Tan TK, Hari R, Koepfli KP, Wee WY, Antunes A, Sitam FT, Rovie-Ryan JJ, Karuppannan KV, Wong GJ, et al. 2016. De novo sequencing, assembly and analysis of eight different transcriptomes from the Malayan pangolin. Sci Rep 6:28199. 
Murthy KG, Deb A, Goonesekera S, Szabo C, Salzman AL. 2004. Identification of conserved domains in Salmonella muenchen flagellin that are essential for its ability to activate TLR5 and to induce an inflammatory response in vitro. The Journal of biological chemistry 279:5667-5675.

Okin D, Medzhitov R. 2012. Evolution of inflammatory diseases. Curr Biol 22:R733-740.

Osipova E, Hecker N, Hiller M. 2019. RepeatFiller newly identifies megabases of aligning repetitive sequences and improves annotations of conserved non-exonic elements. Gigascience 8.

Padilla-Carlin DJ, McMurray DN, Hickey AJ. 2008. The guinea pig as a model of infectious diseases. Comp Med 58:324-340.

Quinlan AR, Hall IM. 2010. BEDTools: a flexible suite of utilities for comparing genomic features. Bioinformatics 26:841-842.

Sackton TB, Lazzaro BP, Schlenke TA, Evans JD, Hultmark D, Clark AG. 2007. Dynamic evolution of the innate immune system in Drosophila. Nature genetics 39:1461-1468.

Sadier A, Davies KT, Yohe LR, Yun K, Donat P, Hedrick BP, Dumont ER, Davalos LM, Rossiter SJ, Sears KE. 2018. Multifactorial processes underlie parallel opsin loss in neotropical bats. Elife 7.

Sharma V, Elghafari A, Hiller M. 2016. Coding exon-structure aware realigner (CESAR) utilizes genome alignments for accurate comparative gene annotation. Nucleic acids research 44:e103.

Sharma V, Hecker N, Roscito JG, Foerster L, Langer BE, Hiller M. 2018a. A genomics approach reveals insights into the importance of gene losses for mammalian adaptations. Nat Commun 9:1215.

Sharma V, Hiller M. 2019. Losses of human disease-associated genes in placental mammals. NAR Genomics and Bioinformatics 2:Iqz012.

Sharma V, Lehmann T, Stuckas H, Funke L, Hiller M. 2018b. Loss of RXFP2 and INSL3 genes in Afrotheria shows that testicular descent is the ancestral condition in placental mammals. PLoS biology 16:e2005293.

Sharma V, Schwede P, Hiller M. 2017. CESAR 2.0 substantially improves speed and accuracy of comparative gene annotation. Bioinformatics 33:3985-3987.

Smith KD, Andersen-Nissen E, Hayashi F, Strobe K, Bergman MA, Barrett SL, Cookson BT, Aderem A. 2003. Toll-like receptor 5 recognizes a conserved site on flagellin required for protofilament formation and bacterial motility. Nat Immunol 4:1247-1253.

Smith SA, Jann OC, Haig D, Russell GC, Werling D, Glass EJ, Emes RD. 2012. Adaptive evolution of Toll-like receptor 5 in domesticated mammals. BMC evolutionary biology 12:122.

Tsujita T, Tsukada H, Nakao M, Oshiumi H, Matsumoto M, Seya T. 2004. Sensing bacterial flagellin by membrane and soluble orthologs of Toll-like receptor 5 in rainbow trout (Onchorhynchus mikiss). The Journal of biological chemistry 279:48588-48597.

Uematsu S, Jang MH, Chevrier N, Guo Z, Kumagai Y, Yamamoto M, Kato H, Sougawa N, Matsui H, Kuwata $\mathrm{H}$, et al. 2006. Detection of pathogenic intestinal bacteria by Toll-like receptor 5 on intestinal CD11c+ lamina propria cells. Nat Immunol 7:868-874.

Velova H, Gutowska-Ding MW, Burt DW, Vinkler M. 2018. Toll-like receptor evolution in birds: gene duplication, pseudogenisation and diversifying selection. Mol Biol Evol 35:2170-2184.

Vijay K. 2018. Toll-like receptors in immunity and inflammatory diseases: Past, present, and future. Int Immunopharmacol 59:391-412. 
Vijay-Kumar M, Aitken JD, Carvalho FA, Cullender TC, Mwangi S, Srinivasan S, Sitaraman SV, Knight R, Ley RE, Gewirtz AT. 2010. Metabolic syndrome and altered gut microbiota in mice lacking Toll-like receptor 5. Science 328:228-231.

Vijay-Kumar M, Aitken JD, Gewirtz AT. 2008. Toll like receptor-5: protecting the gut from enteric microbes. Semin Immunopathol 30:11-21.

Vijay-Kumar M, Sanders CJ, Taylor RT, Kumar A, Aitken JD, Sitaraman SV, Neish AS, Uematsu S, Akira S, Williams IR, et al. 2007. Deletion of TLR5 results in spontaneous colitis in mice. J Clin Invest 117:39093921.

Vinkler M, Bainova H, Bryja J. 2014. Protein evolution of Toll-like receptors 4, 5 and 7 within Galloanserae birds. Genet Sel Evol 46:72.

Voogdt CG, Bouwman LI, Kik MJ, Wagenaar JA, van Putten JP. 2016. Reptile Toll-like receptor 5 unveils adaptive evolution of bacterial flagellin recognition. Sci Rep 6:19046.

Warr A, Affara N, Aken B, Beiki H, Bickhart DM, Billis K, Chow W, Eory L, Finlayson HA, Flicek P, et al. 2019. An improved pig reference genome sequence to enable pig genetics and genomics research. BioRxiv:668921.

Watson M, Warr A. 2019. Errors in long-read assemblies can critically affect protein prediction. Nature biotechnology 37:124-126.

Wertheim JO, Murrell B, Smith MD, Kosakovsky Pond SL, Scheffler K. 2015. RELAX: detecting relaxed selection in a phylogenetic framework. Mol Biol Evol 32:820-832.

West TE, Chantratita N, Chierakul W, Limmathurotsakul D, Wuthiekanun V, Myers ND, Emond MJ, Wurfel MM, Hawn TR, Peacock SJ, et al. 2013. Impaired TLR5 functionality is associated with survival in melioidosis. J Immunol 190:3373-3379.

Wlasiuk G, Khan S, Switzer WM, Nachman MW. 2009. A history of recurrent positive selection at the tolllike receptor 5 in primates. Mol Biol Evol 26:937-949.

Yates AD, Achuthan P, Akanni W, Allen J, Allen J, Alvarez-Jarreta J, Amode MR, Armean IM, Azov AG, Bennett R, et al. 2019. Ensembl 2020. Nucleic acids research.

Youngblut ND, Reischer GH, Walters W, Schuster N, Walzer C, Stalder G, Ley RE, Farnleitner AH. 2019. Host diet and evolutionary history explain different aspects of gut microbiome diversity among vertebrate clades. Nat Commun 10:2200.

Yu Y, Zeng H, Lyons S, Carlson A, Merlin D, Neish AS, Gewirtz AT. 2003. TLR5-mediated activation of p38 MAPK regulates epithelial IL-8 expression via posttranscriptional mechanism. Am J Physiol Gastrointest Liver Physiol 285:G282-290. 


\section{Figures}
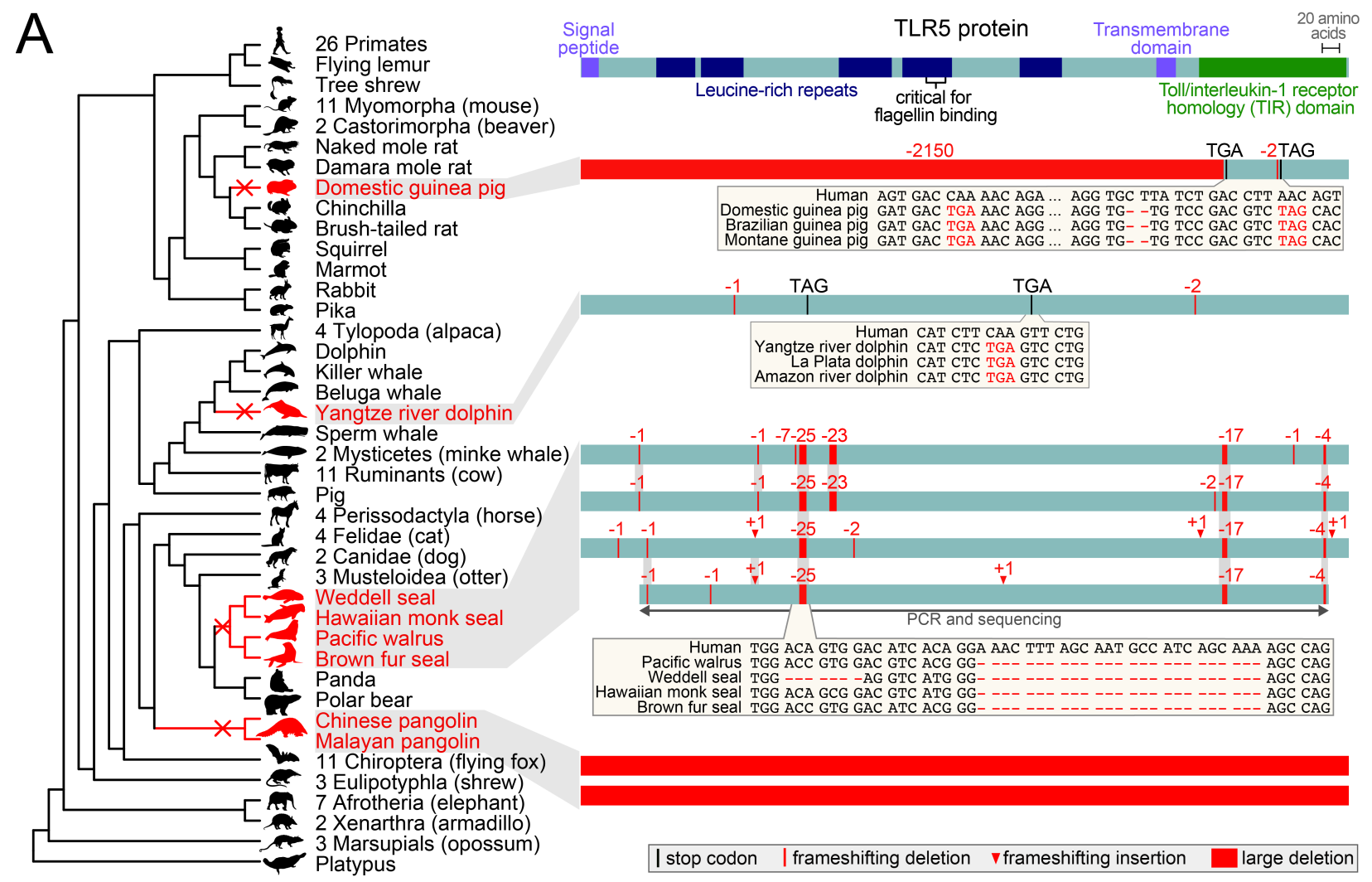

B Human genome (hg38) chr1:223,109,587-223,124,700

GENCODE v32 gene annotation

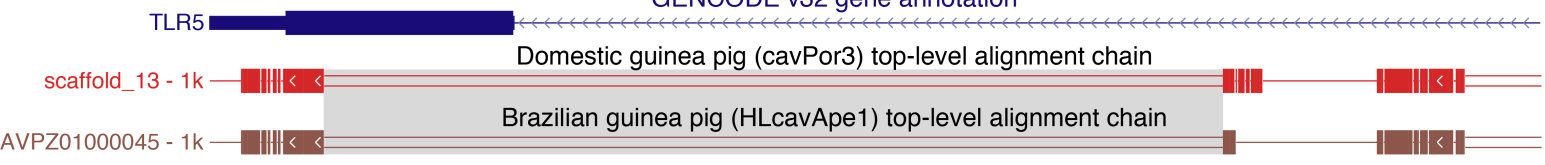

C Human genome (hg38) chr1:223,102,263-223,224,219

$50 \mathrm{~kb}$

GENCODE v32 gene annotation

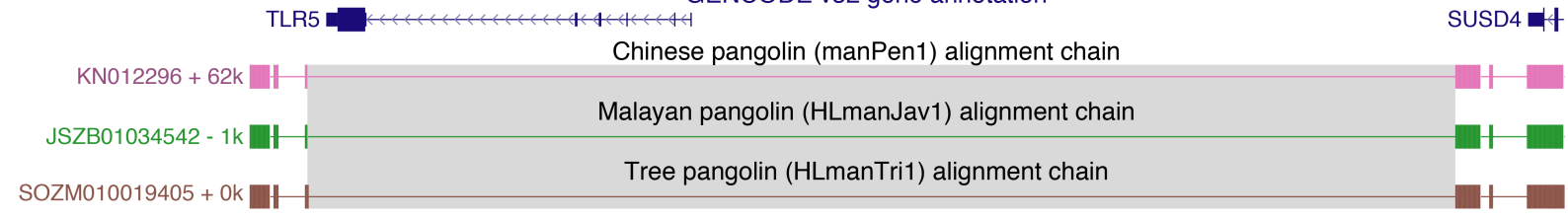

Figure 1: Loss of TLR5 in four independent mammalian lineages.

(A) Left: Phylogeny of mammals, showing species that lost TLR5 in red font. Right: The human TLR5 protein is visualized at the top, superimposed with the signal peptide, transmembrane and Pfam protein domains, as annotated in Ensembl (Yates, et al.), and the region required for flagellin binding (Mizel, et al. 2003). Boxes below represent the $T L R 5$ coding exon of individual species, superimposed with gene-inactivating mutations. For the brown fur seal, we sequenced the majority of the TLR5 exon. Insets show 
inactivating mutations (red font) that are shared among sister species. Mutations shared between at least two pinniped species are highlighted by grey background.

(B) UCSC genome browser (Haeussler, et al. 2019) view of the human hg38 genome, showing the TLR5 gene and top-level chain of co-linear local alignments (blocks represent aligning regions, double lines represent unaligning sequence, and single lines represent deletions) to two guinea pig species. Alignment chains show that the large deletion that removes the majority of the TLR5 coding exon (grey background) has the same breakpoints and thus likely occurred in the ancestor of both guinea pigs.

(C) UCSC genome browser view shows that the TLR5 deletion has breakpoints shared between three pangolin species, suggesting that TLR5 loss already occurred in their common ancestor. 